The authors propose that URB597 blocks intracellular anandamide degradation, increasing interaction of this substance with a subset of brain $\mathrm{CB}_{1}$ receptors and regulating monoaminergic neurotransmission and response to stress. URB597 has this effect without the hedonic, rewarding effects associated with direct-acting cannabinoid agonists. The results, say the authors, highlight fatty-acid amide hydrolase as a new target for antidepressant drugs and provide preclinical validation for the use of URB597 as an antidepressant agent.

Pippa Murdie

Original article Gobbi G et al. (2005) Antidepressantlike activity and modulation of brain monoaminergic transmission by blockade of anandamide hydrolysis. Proc Natl Acad Sci USA 102: 18620-18625

\section{Aspirin use before intracerebral hemorrhage increases mortality risk}

Hematoma volume predicts outcome and mortality risk following intracerebral hemorrhage (ICH). Therapies that decrease coagulability could cause enlargement of hematomas, thereby increasing the risk of mortality in patients with $\mathrm{ICH}$. Saloheimo et al. studied outcomes in $\mathrm{ICH}$ patients regularly taking aspirin or warfarin at presentation.

In this population-based investigation, patients with spontaneous $\mathrm{ICH}$ were identified by head CT scan $(n=203)$ or autopsy $(n=5)$ over a 33-month study course. Regular aspirin use before $\mathrm{ICH}$ was significantly linked with hematoma enlargement in the week immediately following hemorrhage $(P=0.006)$. The 3-month mortality rate in all patients was $33 \%$, and independent risk factors for death were regular aspirin use or warfarin use at onset of $\mathrm{ICH}$ (relative risk 2.5, 95\% $\mathrm{Cl} 1.3-4.6, P=0.004$, and 3.2, $95 \% \mathrm{Cl} 1.6-6.1, P=0.001$, respectively).

The results show that patients who had regularly used aspirin or warfarin before $\mathrm{ICH}$ were at increased risk of death within 3 months of onset compared with nonusers. In aspirin users, this increased risk probably results from early hematoma growth; however, the authors were unable to confirm this hypothesis because only some patients underwent second CT scans. Nevertheless, the results indicate a need to block hematoma growth and to stop anticoagulation in $\mathrm{ICH}$ patients taking regular aspirin or warfarin.
Further work is required to test whether occasional aspirin use affects outcome after $\mathrm{ICH}$, and whether the benefits of interventions that increase platelet function in $\mathrm{ICH}$ patients taking aspirin would outweigh their risks.

Kate Matthews

Original article Saloheimo P et al. (2006) Regular aspirinuse preceding the onset of primary intracerebral hemorrhage is an independent predictor for death. Stroke 37: 129-133

\section{Myocardial infarction is associated with increased risk of subsequent stroke}

The risk factors and treatments for myocardial infarction (MI) and stroke are similar. Investigations have shown that the incidence of $\mathrm{Ml}$ is decreasing and survival after such an event is increasing; however, it is not known whether these changes also affect the incidence of stroke after index MI. Witt and colleagues examined the occurrence of ischemic and hemorrhagic stroke in a community-based cohort study of 2,160 patients who were hospitalized with incident MI within the period 1979-1998.

The median follow-up was 5.6 years (range 0-22 years) and 273 strokes were recorded. The rate of stroke occurring within 30 days of the index $\mathrm{Ml}$ was 22.6 per 1,000 personmonths-a 44-fold increase relative to the expected rate for the general population, calculated from the Rochester Stroke Registry of the same community. In the first 3 years following $\mathrm{MI}$, the incidence of stroke was 2-3 times higher than in the general community, and the risk of stroke was increased with diabetes, increasing age and previous stroke. The risk of stroke after Ml did not decline over the 19-year study period and the occurrence of stroke was associated with increased risk of death (adjusted hazard ratio 2.89 ; 95\% $\mathrm{Cl} 2.44-3.43$ ).

The results show that treatments that have reduced the incidence of $\mathrm{Ml}$ do not decrease the risk of subsequent stroke. The use of antithrombotic interventions to prevent stroke should be considered, particularly in the 3 years immediately after MI. The study cohort was mostly white and, therefore, these findings might not be generalizable to other ethnic populations.

Kate Matthews

Original article Witt BJ et al. (2005) A community-based study of stroke incidence after myocardial infarction. Ann Intern Med 143: 785-792 